\title{
An Extension of Transformation-based Reversible and Quantum Circuit Synthesis
}

\author{
Mathias Soeken \\ Integrated Systems Laboratory \\ EPFL, Switzerland \\ mathias.soeken@epfl.ch
}

\author{
Gerhard W. Dueck, Md. Mazder Rahman \\ Faculty of Computer Science \\ University of New Brunswick, Canada \\ \{gdueck,md._mazder.rahman\}@unb.ca
}

\author{
D. Michael Miller \\ Department of Computer Science \\ University of Victoria, Canada \\ mmiller@uvic.ca
}

\begin{abstract}
Transformation-based synthesis is a well established systematic approach to determine a circuit implementation from a reversible function specification. Due to the inherent bidirectionality of reversible circuits the basic method can be applied in a bidirectional manner. In the approaches to date, gates are added either to the input side or the output side of the circuit on each iteration. In this paper, we introduce a new variation where gates may be added at both ends during a single iteration when this is advantageous to reducing the cost of the circuit. Experimental results show the advantage of the new approach over previous transformation-based synthesis methods and that the additional computation is justified by the possibility of improved circuit costs.
\end{abstract}

\section{INTRODUCTION}

Reversible circuits have been widely studied because of their potential for reducing power consumption [1] and because of their connection to quantum computation [2]. A key issue is the synthesis of reversible circuits. This is a difficult problem that is significantly different from the synthesis of circuits employing traditional irreversible gates. Exact synthesis is only possible for very small circuits. The methods discussed here are heuristic and greedy relying on local optimization.

Transformation-based reversible circuit synthesis was introduced in [3] where two algorithms, a basic (unidirectional) approach and a bidirectional extension, were given. The bidirectional approach exploits the reversibility of both gates and circuits and while the basic algorithm builds a circuit in only one direction, typically from output to input, the bidirectional approach builds the circuit from both the output and the input sides. The bidirectional approach outperforms the basic algorithm

In this paper, we introduce a new generalized approach which as experimental results will show can yield significantly smaller circuits in some cases. On each iteration, the bidirectional algorithm adds gates to either the input or the output side of the circuit, but not both. The new algorithm better exploits the structure of the reversible function being synthesized and can add gates at both ends on each iteration when that is of advantage.

\section{BACKGROUND}

A multiple-output Boolean function is reversible if it maps each input assignment to a unique output assignment, i.e. it is a bijection; otherwise the function is irreversible. A reversible function can be realized by a cascade of reversible gates with no fan-out or feedback [2]. A completely or incompletelyspecified irreversible function can be embedded into a re- versible function, usually with more inputs (constants) and outputs (garbage), and then realized by a reversible circuit [4].

A multiple-control Toffoli (MCT) gate with target line $x_{j}$ and control lines $\left\{x_{i_{1}}, x_{i_{2}}, \ldots, x_{i_{k}}\right\}$, maps $\left(x_{1} \ldots x_{j} \ldots x_{n}\right)$ to $\left(x_{1} \ldots\left(x_{i_{1}} x_{i_{2}} \cdots x_{i_{k}}\right) \oplus x_{j} \ldots x_{n}\right)$. Note that all controls must be 1 (positive) for the target to be inverted. An MCT gate with no control is a NOT gate. An MCT gate with a single control line is called a controlled-NOT (CNOT) gate. Note that while we do not consider the option of negative controls (i.e. controls sensitive to 0 rather than 1 ), the methods discussed here can be extended to allow them. Also, while in this paper we concentrate on MCT gates, the approach can be modified to allow other reversible gates such as Fredkin [5], [6], Peres and inverse-Peres gates [7].

Many quantum gates have been defined and studied in the literature [2]. In this paper, we concentrate on the following gates which we assume have unit cost: NOT and CNOT (as defined above); the 2-line controlled- $V$ gate which changes the target line using the transformation defined by the matrix $\mathbf{V}=\frac{1+i}{2}\left(\begin{array}{cc}1 & -i \\ -i & 1\end{array}\right)$ if the single control line has the value 1; and the 2-line controlled- $V^{+}$gate which changes the target line using the transformation $\mathbf{V}^{+}=\mathbf{V}^{-1}=\frac{1-i}{2}\left(\begin{array}{ll}1 & i \\ i & 1\end{array}\right)$ if the single control line has the value 1 . Gates $V$ and $V^{+}$are referred to as controlled-square-root-of-NOT gates since $\mathbf{V}^{2}=\left(\mathbf{V}^{+}\right)^{2}=$ $\left(\begin{array}{ll}0 & 1 \\ 1 & 0\end{array}\right)$

A circuit line not used as the target or as a control of a gate is an ancillary line for that gate. Such lines are used in realizing an MCT gate using quantum gates, see [8], [9]

\section{TRANSFORMATION-BASED SYNTHESIS ALGORITHMS}

In this paper for ease of description, we present transformation-based synthesis in terms of the truth table representation of a reversible function. Note that transformationbased methods can be implemented using more efficient representations such as decision diagrams [10].

\section{A. Basic Algorithm}

Given a truth table representing a reversible function $f$, the basic transformation-based synthesis algorithm [3] proceeds through the truth table rows in order $0 \leq i<2^{n}-1$. At each row $i$, if $f(i) \neq i$ MCT gates are selected to map $f(i)$ to $i$. These gates are chosen such that they do not affect any row $j$ for $j<i$, i.e. those that have already been considered. The gates are added to the circuit being constructed from the output towards the input and the reversible specification is updated by applying the gates to the output side of the specification. When 
all rows $0 \leq i<2^{n}-1$ have been considered the resulting truth table is the identity function and the gates chosen represent an implementation of the original reversible function. Note that row $2^{n}-1$ does not have to be considered as $f\left(2^{n}-1\right)=2^{n}-1$ after all previous rows are made to match.

\section{B. Bidirectional Algorithm}

The bidirectional transformation-based synthesis which is an extension of the basic algorithm was also introduced in [3]. For each row $i$, the gates $G_{0}$ required to transform the output pattern $f(i)$ to $i$ are determined as in the basic algorithm. In addition, there must be a row $j$ later in the table where $f(j)=i$. MCT gates $G_{1}$ that transform $j$ to $i$ are determined. The less expensive of $G_{0}$ and $G_{1}$ is determined and those gates are added to the circuit and used to update $f$. Note that if $G_{1}$ is chosen the gates apply from the input toward the output of the circuit and are used to update the input side of the specification. The cost of a set of gates can be simply the MCT gate count or can be based on the quantum cost of implementing the MCT gates. We use the latter in this work.

\section{A New Algorithm}

Our new method is shown in Algorithm 1. For each row $i$, the new generalized algorithm tries every row from $i$ to $2^{n}-1$ mapping the input and the output patterns to $i$ thereby potentially adding gates to both the input and the output side of the circuit. The algorithm chooses the first such mapping that has lowest cost. To see the new algorithm subsumes the previous two, note that the basic algorithm is the case of only considering $j=i$, while the bidirectional algorithm is the case of only considering two cases: $j=i$ and $j$ such that $f(j)=i$.

Algorithm 1 employs three functions: $\operatorname{Cost}(G)$ which returns the quantum cost of a list of MCT gates $G$; RMdist $(f)$ which determines the Hamming distance between the ReedMuller spectra [11] of a reversible function $f$ and the identity function; and $\operatorname{Map}(y, x)$ which returns a set of MCT gates that map the pattern $y$ to $x$ where $y>x$ such that the gates to do not affect any $z<x$.

In this work, we use a simple version of $\operatorname{Cost}(G)$ that sums the quantum cost of each MCT gate in $G$ using the MCT quantum gates costs from [12]. It does not look for simplifications between MCT gates.

RMdist $(f)$ computes the Hamming distance between the Reed-Muller spectrum of $f$ and the Reed-Muller spectrum of the identity function with the same number of inputs and outputs as $f$. The identity function is the reversible function that maps each input pattern to itself. The Hamming distance between two spectra is the sum of the Hamming distances between the corresponding output patterns. The Reed-Muller spectrum is used since it measures global rather than local function information. The Hamming distance is used to measure how close two spectra are to each other.

$\operatorname{Map}(y, x)$ is described in Algorithm 2. It identifies a sequence of MCT gates to map the bit pattern $y$ to $x$ where $y>x$ which is a consequence of going through the rows of the truth table in ascending order. As noted, the gates are selected so that they have no effect on any bit pattern $z<x$. Map uses a function MakeGate(target, controls) that creates the representation of an MCT gate with specified target and controls.
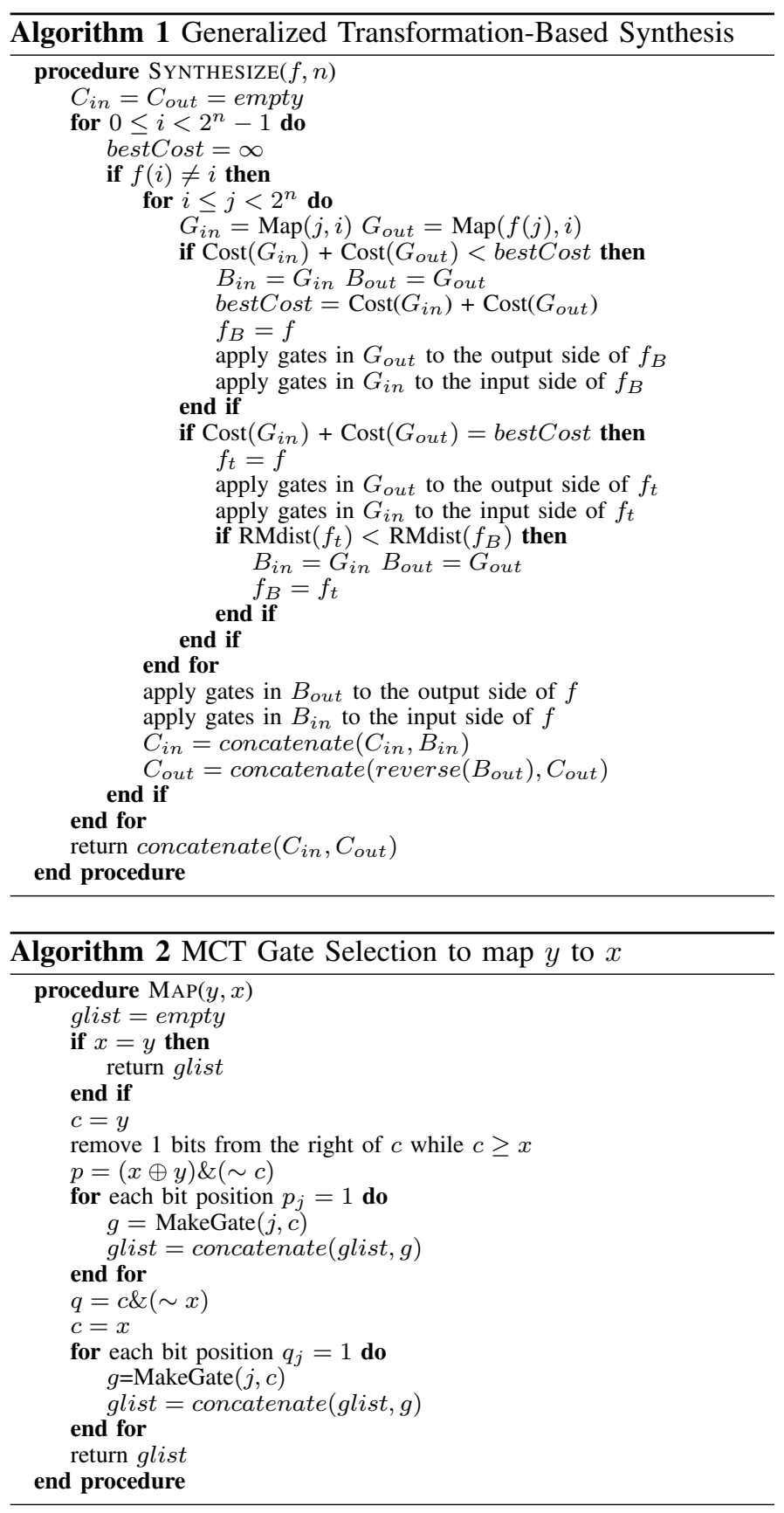

Algorithm 2 begins by setting the control specification $c$ to have as few 1's as possible from $y$ such that $c \geq x$. The latter condition is required to be sure the gates will not affect earlier rows in the truth table. The first for loop generates MCT gates with controls $c$ with one gate for each variable outside $c$ that has to be flipped to make $y$ match $x$. The second for loop then uses $x$ as the control and generates one gate for each variable in $c$ that has to be made 0 to match $x$. In both loops each gate generated has a variable whose value is to change as its target.

\section{EXPERIMENTAL RESULTS}

We have implemented the basic, bidirectional and new synthesis algorithms in Python which is convenient as it has efficient built-in list handling and reversible and quantum circuits are lists of gates. Table I shows the results of applying each 
TABLE I. 3 VARIABLE FUnCTIONS

\begin{tabular}{|l|c|c|c|}
\hline & $\begin{array}{c}\text { Basic } \\
\text { Algorithm }\end{array}$ & $\begin{array}{c}\text { Bidirectional } \\
\text { Algorithm }\end{array}$ & $\begin{array}{c}\text { New } \\
\text { Algorithm }\end{array}$ \\
\hline Max MCT Gates & 17 & 15 & 14 \\
Avg. MCT Gates & 8.67 & 7.06 & 6.85 \\
Max NCV Gates & 29 & 29 & 29 \\
Avg. NCV Gates & 17.87 & 16.16 & 15.72 \\
\hline
\end{tabular}

of the algorithms to all 40,320 3-variable reversible functions. The bidirectional method reduces the average numbers of MCT and NCV gates required and the new algorithm reduces the averages further. The differences are not particularly large because there is little scope for optimization with 3 variables.

Table II shows the results of applying transformation-based synthesis for a selection of benchmarks of varying size taken from RevLib [13]. The first section of the table gives the name and the number of circuit lines for each benchmark The next three sections show the number of MCT gates in the circuits produced by the basic, the bidirectional and the new transformation-based synthesis methods respectively (labeled Synth.), and the number of gates after application of MCT template reduction [14] (labeled Opt.). Note that in this paper we restrict our attention to circuits with positive controls. Further reduction can be achieved if negative controls are used, assuming they are permitted in the target technology.

The lowest synthesized and optimized gate counts for each benchmark are indicated in bold. The final section of the table shows the percentage improvements (a few are negative) for the new algorithm compared to the better of the results from the basic and the bidirectional algorithms. Percentages are shown for the synthesized and the optimized gate counts.

The techniques presented including the new generalized transformation-based synthesis algorithm employ heuristic and greedy techniques. Hence no approach is best in all cases and is the reason for the negative improvements in Tables II. However these results do illustrate the potential benefit of the new algorithm and show that our new approach is generally as good or better than the unidirectional and bidirectional transformation approaches. Several of the benchmarks show that the advantage can be very significant so that the new algorithm can be considered an important addition to previous transformation based approaches.

Table III shows results for mapping the MCT circuits summarized in Table II to NCV circuits. The mapping method developed by Sasanian [15] was used first with the NCV gate counts shown as Map. A * beside the gate count means an extra helper line had to be added to the circuit during the MCT to NCV mapping. This occurs when there is at least one MCT gate that uses every line in the circuit.

The NCV circuits were then optimized using NCV template matching [14] yielding smaller circuits with the gate counts shown as Opt. Minimums are shown in bold and the percentage improvements are shown as before. Once again, because the methods are heuristic and because the optimization depends on the actual circuit structure, some unexpected results arise. For example, for benchmark hwb5 21 the NCV circuit is largest for the basic algorithm, but after optimization it becomes the smallest of the three options. However, in general, except for the hwb (hidden weight bit) functions and ckt3_cycle_68 mapping and optimizing the circuits from the new algorithm gives the best results and in some cases they are significantly better. We are studying the structure of the hwb functions and ckt 3 in an effort to improve the new algorithm.

\section{CONCLUSION}

This paper has presented a new approach to transformationbased synthesis of reversible and quantum circuits. The experimental results show this approach can in some cases give significantly better circuits than do the basic and the bidirectional transformation-based algorithms.

It is clear from Algorithms 1 and 2 that the overall procedure has complexity $O\left(n 2^{2 n}\right)$ whereas the basic transformation-based synthesis procedure has complexity $O\left(n 2^{n}\right)$. The space requirement is the more restrictive parameter for truth-table based approaches and is the same, $O\left(2^{n}\right)$, for all three synthesis approaches considered. As noted earlier, this can be improved by using decision diagrams.

Ongoing work will consider variants of the Map function in order to further reduce the quantum cost of the synthesized circuits. We plan to consider implementation of the method using symbolic function representations rather then truth tables and to examine the extension of the method to partiallyspecified reversible functions. We will also consider how the concepts in [5] apply to our method.

Acknowledgements: This work was partially supported by NSERC Discovery Grants. We thank Dr. Z. Sasanian for the use of her NCV circuit optimization program and the reviewers for their helpful comments.

\section{REFERENCES}

[1] C. H. Bennett, "Logical reversibility of computation," IBM J. Research and Development, vol. 17, no. 6, pp. 525-532, 1973.

[2] M. Nielsen and I. Chuang, Quantum Computation and Quantum Information. Cambridge Univ. Press, 2000.

[3] D. M. Miller, D. Maslov, and G. W. Dueck, "A transformation based algorithm for reversible logic synthesis," in Proc. Design Automation Conf., 2003, pp. 318-323.

[4] M. Soeken, R. Wille, O. Keszocze, D. M. Miller, and R. Drechsler, "Embedding of large Boolean functions for reversible logic," ACM J. on Emerging Technologies in computing Systems, vol. 12, 2015.

[5] C. Chandak, A. Chattopadhyay, and S. M. S. Maitra, "Analysis and improvement of transformation-based reversible logic synthesis," in Proc. Int'l Symp. on Multiple-valued Logic, 2013, pp. 47-52.

[6] M. Soeken and A. Chattopadhyay, "Fredkin-enabled transformationbased reversible logic synthesis," in Proc. Int'l Symp. on Multiplevalued Logic, 2015, pp. 60-65.

[7] A. Peres, "Reversible logic and quantum computers," Physical Review A, vol. 32, no. 6, pp. 3266-3276, 1985.

[8] A. Barenco, C. H. Bennett, R. Cleve, D. P. DiVincenzo, M. Margolus, P. Shor, T. Sleator, J. A. Smolin, and H. Weinfurter, "Elementary gates for quantum computation," Physical Review A, vol. 52, no. 5, pp. 34573467, 1995.

[9] D. M. Miller, "Lower cost quantum gate realizations of multiple-control Toffoli gates," in IEEE Pacific Rim Conference, 2009, pp. 308-313.

[10] M. Soeken, L. Tague, G. W. Dueck, and R. Drechsler, "Ancilla-free synthesis of large reversible functions using binary decision diagrams," J. Symb. Comput., vol. 73, pp. 1-26, 2016.

[11] D. Muller, "Application of Boolean algebra to switching circuit design and error detection," IRE Trans., vol. 1, pp. 6-12, 1954.

[12] Z. Sasanian and D. M. Miller, "Mapping a multiple-control Toffoli gate cascade to an elementary quantum gate circuit," J. of Multiple-valued Logic and Soft Computing, vol. 18, no. 1, pp. 83-98, 2012.

[13] R. Wille, D. Große, L. Teuber, G. W. Dueck, and R. Drechsler, "RevLib: An online resource for reversible functions and reversible circuits," in Int'l Symp. on Multi-Valued Logic, 2008, pp. 220-225, RevLib is available at www.revlib.org.

[14] M. M. Rahman, "Synthesis of reversible logic," Ph.D. dissertation, University of New Brunswick, 2014.

[15] Z. Sasanian, "Technology mapping and optimization for reversible and quantum circuits," Ph.D. dissertation, University of Victoria, 2012 
TABLE II. MCT EXPERIMENTAL RESULTS

\begin{tabular}{|c|c|c|c|c|c|c|c|c|c|}
\hline \multirow[b]{2}{*}{ Benchmark } & \multirow[b]{2}{*}{ Lines } & \multicolumn{2}{|c|}{$\begin{array}{c}\text { Basic } \\
\text { Algorithm }\end{array}$} & \multicolumn{2}{|c|}{$\begin{array}{l}\text { Bidirectional } \\
\text { Algorithm }\end{array}$} & \multicolumn{2}{|c|}{$\begin{array}{c}\text { New } \\
\text { Algorithm }\end{array}$} & \multirow[b]{2}{*}{ Synth. \% } & \multirow[b]{2}{*}{ Opt. \% } \\
\hline & & Synth. & Opt. & Synth. & Opt. & Synth. & Opt. & & \\
\hline $317 \quad 6$ & 3 & 14 & 11 & 7 & 7 & 7 & 7 & $0.0 \%$ & $0.0 \%$ \\
\hline fredkin 3 & 3 & 3 & 3 & 3 & 3 & 3 & 3 & $0.0 \%$ & $0.0 \%$ \\
\hline ham3_47 & 3 & 6 & 5 & 6 & 5 & 6 & 5 & $0.0 \%$ & $0.0 \%$ \\
\hline miller_5 & 3 & 6 & 6 & 6 & 6 & 5 & 5 & $16.7 \%$ & $16.7 \%$ \\
\hline peres__4 & 3 & 2 & 2 & 2 & 2 & 2 & 2 & $0.0 \%$ & $0.0 \%$ \\
\hline $4 \_49-7$ & 4 & 30 & 24 & 20 & 19 & 20 & 20 & $0.0 \%$ & $-5.3 \%$ \\
\hline hwb4_20 & 4 & 22 & 22 & 16 & 16 & 13 & 12 & $18.8 \%$ & $25.0 \%$ \\
\hline hwb5_21 & 5 & 60 & 51 & 49 & 47 & 49 & 47 & $0.0 \%$ & $0.0 \%$ \\
\hline $\bmod 5 \mathrm{~d} 1 \ldots 24$ & 5 & 8 & 8 & 8 & 8 & 8 & 8 & $0.0 \%$ & $0.0 \%$ \\
\hline $\bmod 5 \mathrm{~d} 2 \_25$ & 5 & 17 & 14 & 9 & 9 & 9 & 9 & $0.0 \%$ & $0.0 \%$ \\
\hline mod5mils_26 & 5 & 9 & 8 & 5 & 5 & 5 & 5 & $0.0 \%$ & $0.0 \%$ \\
\hline graycode6_19 & 6 & 5 & 5 & 5 & 5 & 5 & 5 & $0.0 \%$ & $0.0 \%$ \\
\hline hwb6__22 & 6 & 147 & 134 & 102 & 97 & 108 & 101 & $-5.9 \%$ & $-4.1 \%$ \\
\hline mod5adder 56 & 6 & 39 & 39 & 28 & 28 & 28 & 28 & $0.0 \%$ & $0.0 \%$ \\
\hline ham7_48 & 7 & 286 & 254 & 98 & 88 & 59 & 53 & $39.8 \%$ & $39.8 \%$ \\
\hline hwb7_23 & 7 & 376 & 345 & 346 & 307 & 316 & 286 & $8.7 \%$ & $6.8 \%$ \\
\hline ckt2_cycle_67 & 8 & 890 & 787 & 709 & 640 & 685 & 642 & $3.4 \%$ & $-0.3 \%$ \\
\hline hwb8_54 & 8 & 925 & 822 & 710 & 647 & 674 & 627 & $5.1 \%$ & $3.1 \%$ \\
\hline ckt1_cycle_66 & 9 & 1966 & 1746 & 1680 & 1509 & 1619 & 1480 & $3.6 \%$ & $1.9 \%$ \\
\hline ckt5_cycle_69 & 9 & 1318 & 1173 & 909 & 843 & 845 & 774 & $7.0 \%$ & $8.2 \%$ \\
\hline hwb 55 & 9 & 2078 & 1851 & 1802 & 1623 & 1763 & 1604 & $2.2 \%$ & $1.2 \%$ \\
\hline ckt3_cycle_68 & 10 & 4038 & 3545 & 3519 & 3162 & 3460 & 3156 & $1.7 \%$ & $0.2 \%$ \\
\hline cycle10_2_51 & 12 & 19 & 19 & 19 & 19 & 19 & 19 & $0.0 \%$ & $0.0 \%$ \\
\hline plus63mod4096_72 & 12 & 394 & 392 & 394 & 392 & 18 & 18 & $95.4 \%$ & $95.4 \%$ \\
\hline plus127mod8192_71 & 13 & 781 & 779 & 781 & 779 & 19 & 19 & $97.6 \%$ & $97.6 \%$ \\
\hline plus63mod8192_73 & 13 & 457 & 455 & 457 & 455 & 20 & 20 & $95.6 \%$ & $95.6 \%$ \\
\hline $04101+B 7: C 33+B 7: C 3384 \_77$ & 14 & 48 & 48 & 37 & 37 & 37 & 37 & $0.0 \%$ & $0.0 \%$ \\
\hline
\end{tabular}

TABLE III. NCV EXPERIMENTAL RESULTS

\begin{tabular}{|c|c|c|c|c|c|c|c|c|}
\hline \multirow[b]{2}{*}{ Benchmarl $\backslash \mathbf{k}$} & \multicolumn{2}{|c|}{$\begin{array}{c}\text { Basic } \\
\text { Algorithm }\end{array}$} & \multicolumn{2}{|c|}{$\begin{array}{l}\text { Bidirectional } \\
\text { Algorithm }\end{array}$} & \multicolumn{2}{|c|}{$\begin{array}{c}\text { New } \\
\text { Algorithm }\end{array}$} & \multirow[b]{2}{*}{ Мap \% } & \multirow[b]{2}{*}{ Opt\% } \\
\hline & Map & Opt. & Map & Opt. & Map & Opt. & & \\
\hline $3 \_17 \_6$ & 22 & 12 & 13 & 11 & 13 & 11 & $0.0 \%$ & $0.0 \%$ \\
\hline fredkin_3 3 & 14 & 7 & 14 & 7 & 14 & 7 & $0.0 \%$ & $0.0 \%$ \\
\hline ham $3 \_\overline{47}$ & 9 & 7 & 9 & 7 & 9 & 7 & $0.0 \%$ & $0.0 \%$ \\
\hline miller_5 & 9 & 8 & 9 & 8 & 9 & 8 & $0.0 \%$ & $0.0 \%$ \\
\hline peres_4 & 4 & 4 & 4 & 4 & 4 & 4 & $0.0 \%$ & $0.0 \%$ \\
\hline $4 \_49-7$ & $69^{*}$ & 48 & $80 *$ & 61 & $68 *$ & 41 & $1.4 \%$ & $14.6 \%$ \\
\hline hwwb4_20 & $67 *$ & 52 & $50 *$ & 37 & 22 & 20 & $56.0 \%$ & $45.9 \%$ \\
\hline hwb5_21 & $308^{*}$ & 207 & $284 *$ & 228 & $284 *$ & 228 & $0.0 \%$ & $-10.1 \%$ \\
\hline $\bmod 5 \overline{\mathrm{d} 1} \quad 24$ & 11 & 9 & 11 & 9 & 11 & 9 & $0.0 \%$ & $0.0 \%$ \\
\hline $\bmod 5 \mathrm{~d} 2-25$ & 18 & 15 & 14 & 11 & 14 & 11 & $0.0 \%$ & $0.0 \%$ \\
\hline $\bmod 5$ mils_26 26 & 10 & 9 & 9 & 9 & 9 & 9 & $0.0 \%$ & $0.0 \%$ \\
\hline graycode $6-19$ & 5 & 5 & 5 & 5 & 5 & 5 & $0.0 \%$ & $0.0 \%$ \\
\hline hwb6_22 & $957 *$ & 722 & 580* & 372 & $672 *$ & 472 & $-15.9 \%$ & $-26.9 \%$ \\
\hline mod5adder_56 & $402 *$ & 349 & 237 & 235 & 237 & 235 & $0.0 \%$ & $0.0 \%$ \\
\hline ham7__48 & $2241 *$ & 1703 & 568 & 554 & 286 & 270 & $49.6 \%$ & $51.3 \%$ \\
\hline hwb7_23 & $3132 *$ & 2517 & $2773 *$ & 2087 & 2759* & 2088 & $0.5 \%$ & $0.0 \%$ \\
\hline ckt2_cycle_67 & $8527 *$ & 7009 & $7222 *$ & 5696 & $6895^{*}$ & 5413 & $4.5 \%$ & $5.0 \%$ \\
\hline hwb 8 & $8846^{*}$ & 7409 & $6469 *$ & 5049 & 7222 & 5696 & $-11.6 \%$ & $-12.8 \%$ \\
\hline ckt1_cycle_66 & $21261 *$ & 17948 & $18703 *$ & 15442 & 18402 & 15132 & $1.6 \%$ & $2.0 \%$ \\
\hline ckt5_cycle_69 & $16615^{*}$ & 13939 & $13170 *$ & 10716 & 11685* & 9419 & $11.3 \%$ & $12.1 \%$ \\
\hline hwb 955 & $21283^{*}$ & 17869 & $19557^{*}$ & 16080 & 19364* & 16248 & $1.0 \%$ & $-1.0 \%$ \\
\hline ckt3_cycle_68 & $49063^{*}$ & 41935 & 44114* & 37280 & 46411 & 40065 & $-5.2 \%$ & $-7.5 \%$ \\
\hline cycle10_2_51 & 724 & 724 & 724 & 724 & 724 & 724 & $0.0 \%$ & $0.0 \%$ \\
\hline plus63mod4096_72 & $1556^{*}$ & 1283 & $1556^{*}$ & 1283 & $601 *$ & 464 & $61.4 \%$ & $63.8 \%$ \\
\hline plus $127 \bmod 819 \overline{2} \_71$ & $2068 *$ & 1726 & $2068 *$ & 1726 & 726* & 607 & $64.9 \%$ & $64.8 \%$ \\
\hline plus63mod8192_ 73 & $2012 *$ & 1716 & $2012 *$ & 1716 & 751* & 634 & $62.7 \%$ & $63.1 \%$ \\
\hline 04101+B7:C33+B7:C3384_77 & 851 & 845 & 560 & 554 & 560 & 554 & $0.0 \%$ & $0.0 \%$ \\
\hline
\end{tabular}

